\title{
METAPHORISCHE GESCHICHTENINTERAKTION
}

\section{DIE TAUBE IM KÄFIG DES KÁLMÁN MIKSZÁTH}

\author{
PÉTER HAJDU \\ Institut für Literaturwissenschaft, Budapest, \\ Ungarn
}

Seit es durch die Untersuchungen der Metapher klar und allgemein anerkannt wurde, daß die Metapher keine Erscheinung ist, die in den Grenzen eines einzigen Wortes erklärt werden kann, ${ }^{1}$ ist es theoretisch möglich, größere Textabschnitte oder ganze Texte metaphorisch zu interpretieren. Solche Interpretationsversuche sind trotzdem selten, obwohl ein solches Verfahren nicht nur für die Textinterpretation, sondern auch für die Metapherforschung fruchtbar sein könnte. Wie kann aber ein einziger Text metaphorisch interpretiert werden, wenn die Metapher eine Identifizierung zwei verschiedener Sachen ist und folglich zwei verschiedene Sachen voraussetzt? Ich sehe zwei theoretische Möglichkeiten. 1. Der Text ist die erste Sache, die zweite muß sich anderswo finden. Diese Erscheinung wäre in Zusammenhang mit der Metapher nicht überraschend, und auf Grund der Interaktionstheorie müßte eine solche Auffassung nicht bedeuten, daß der Text in Stelle eines - etwa diskursiven - Inhalts dasteht. 2. Man kann beide Elemente und vielleicht auch die Geste der Identifikation im Text selbst auffinden. Ich glaube, daß beide Möglichkeiten mit Nutzen ausgearbeitet werden können; ich werde mich im folgenden nur mit der zweiten - am Beispiel eines Textes, der die zwei Elemente und die Geste der Identifikation offensichtlich innehat - beschäftigen.

Kálmán Mikszáth experimentierte sehr gerne mit dem Nebeneinanderstellen zwei verschiedener Geschichten. Es geht nicht um zwei parallel vorwärtsgehende Geschichten die abwechselnd erzählt werden. Die Geschichten kommen nacheinander: wenn die erste beendet ist, beginnt die zweite. Diese Form kann natürlich ganz didaktisch sein, wenn beide Geschichten von einem einzigen, konsistenten Wertsystem beherrscht werden und die Gegenüberstellung der Geschichten dieses System demonstriert. ${ }^{2}$ Manchmal sind solche Kurzgeschichten Mikszáths wirklich didaktisch, aber durchaus nicht alle. Besonders nicht im Falle seines anspruchsvollsten Versuches dieser Art, Die Taube im Käfig. Wir haben hier zwei Geschichten, die durch Untertitel (Die erste Erzählung und Die zweite Erzählung) sind. Die erste Geschichte spielt vierhundert Jahre früher in Italien, die zweite zu des Autors Lebzeiten in Budapest. In beiden treten zwei Freunde und ein Mädchen 
auf, aber kaum könnten wir auch nur ein einziges gemeinsames Handlungselement in ihnen finden. Trotzdem lesen wir am Ende:

Ich muß nur eins verraten, denn jeder rechtschaffene Schriftsteller spart sich irgendeine Überraschung für den Schluß seiner Erzählung auf. Übrigens machte ich schon zu Beginn derselben kein Geheimnis daraus.

Der Leser meint nämlich, er habe zwei Erzählungen gelesen. Und dies ist die Überraschung. Denn er hat nur eine gelesen, aber zweimal hinter einander. (S. 110) ${ }^{3}$

Es erinnert einen an die Metapher. Nennen wir denn nicht genau das eine Metapher, wenn jemand von zwei offensichtlich verschiedenen Sachen behauptet, sie wären identisch? Es gibt aber einen scheinbar wichtigen Unterschied. Im Fall der Metapher wird von einer Sache gesagt, daß sie mit einer anderen identisch ist, und diese Relation ist irreversibel. Richards hat diese Teile der Metapher tenor und vehicle genannt. ${ }^{4}$ Wir können diese Teile identifizieren und dürfen sie nicht verwechseln. Von den beiden Erzählungen von Die Taube im Käfig wäre es unmöglich zu sagen, welche der Tenor und welche das Vehikel seien. Beide können beide Rollen spielen. Es sei aber daran erinnert, daß nicht einmal die bedeutungsstiftende Funktion der Metapher einbahnig ist. Wenn wir sagen: der Mensch ist ein Wolf, erscheint nicht nur der Mensch in neuem Licht, sondern auch der Wolf als nunmehr anthropomorphes Wesen. ${ }^{5}$ Wenn wir diese Interaktion in der Wirkung der Metapher beobachten, werden wir mit Recht auch solche Identifizierungen Metaphern nennen, in denen Tenor und Vehikel untrennbar sind, andrerseits nähern wir uns der Bachtinschen Theorie des Dialogischen an. Bachtin meint, „das, was die sprachliche Definition eines Gegenstandes erschwere, sei, daß sich in ihm dialogische Wechselwirkungen abspielen, die auf Momente der über den besagten Gegenstand sich heranbildenden sozialen und sprachlichen Bewußtma-

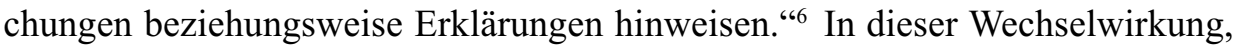
das heißt, bei jedem Gegenstand, setzen die verschiedenen Sprachen den Dialog fort.

Das Metaphorische und das Dialogische sehen wie verwandte Begriffe aus, besonders dann, wenn es um die Interaktion von zwei angeblich identischen Geschichten geht. Nach der Konzeption von Ankersmit ${ }^{7}$ enthält die Metapher gleichzeitig ein deskriptives Element und einen Vorschlag zu einem Gesichtspunkt. Einerseits beschreibt sie den Tenor, andrerseits macht sie einen Vorschlag, wovon und wie wir ihn ansehen sollten, um sein Wesen ergreifen zu können. Mittels der Kategorien Ähnlichkeit und Unterschied können beide Faktoren vielleicht noch besser getrennt werden: die Beschreibung muß sich auf die Ähnlichkeiten gründen, während der neue Gesichtspunkt von den Unterschieden eingegeben wird. Das würde im Fall zweier Geschichten bedeuten, daß wir auf Grund der Ähnlich- 
keiten die sozusagen virtuelle Originalgeschichte rekonstruieren oder besser konstruieren können, deren die zwei Geschichten zwei Verwirklichungen zu sein scheinen, während die Unterschiede die Positionen zeigen, von denen aus die Geschichten angesehen werden sollen. Das heißt, die eine Geschichte soll von der zweiten her gelesen werden und umgekehrt.

Diese Doppelansicht kommt auch in der Position des Erzählers zum Ausdruck. Sooft er die Ereignisse kommentiert, tut er es aus Sicht der jeweiligen anderen Geschichte, diese reflektierend, und manchmal so, daß er einfach Fragen stellt im Namen eines hypothetischen Publikums. Es gibt keinen fixierten autorischen Gesichtspunkt, welcher sich über beiden Geschichten befinden würde und beide Geschichten auf Grund eines homogenen Wertsystems schätzen könnte.

Die Anschauungsweise einer Geschichte, wenn ich so sagen darf, kann von der Anschauungsweise der Erzählung getrennt werden, welche durch die Kommentare des wahrnehmbaren Erzählers auch die Anschauungsweise derselben enthält. Die Ereignisse und die Gestalten - fast an sich, präsentiert von einem unwahrnehmbaren Erzähler - bilden eine Welt, auf die sich der wahrnehmbar gewordene Erzähler beziehen, und der er seine eigene Anschauungsweise (wenn er eine solche hat) gegenüberstellen kann. Die Welt der Geschichte ist folglich dadurch definiert, welche Gestalten und Ereignisse in ihr vorkommen können. Die Welt schreibt auch eine Anschauungsweise vor, weil diese Welt fremd den Erscheinungen gegnübersteht, die dort nicht vorkommen können. (Wie eine Sprache durch die Dinge beschrieben werden kann, für die sie keine Begriffe hat.) Diese Fremdheit kann auf verschiedenen Stufen erscheinen, die aber von dem Ereignispotential der eigenen Welt und dem an ihr gemessenen Unterschied determiniert sind. Die ethischen Wertsysteme sind mit diesen Anschauungsweisen nicht identisch, sondern bilden deren Teile. Im Falle von Die Taube im Käfig identifizieren sich die Erzähler mit den Wertsystemen der Geschichten nicht, obwohl beide Geschichten ihre als immanent dargestellten Wertsysteme haben, wenn auch die zweite ein recht heterogenes.

Meines Erachtens gibt es keinen einzigen Erzähler mit einer absoluten Anschauung, an der er beide Geschichten einzeln messen könnte, sondern er adoptiert beide Male die Anschauungsweise der jeweiligen anderen Geschichte. Es heißt, die Geschichten lesen einander und der Erzähler versucht nur, diese Leseprozesse zu kanalisieren oder sichtbar zu machen. Die Rezeption war nur gegenüber einer Richtung dieser Wechselwirkung empfindlich, insofern sie das Werk als Gesellschaftskritik betrachtete. Es ist nur die zweite Geschichte von der ersten her gelesen, ohne die erste zu relativieren. Natürlich ist der Prozeß, in dem die für den Leser noch unbekannte zweite Geschichte die erste liest, viel schwerer zu realisieren und deshalb auch schwerer wahrzunehmen. Die zweite Geschichte mit der ersten, schon bekannten Geschichte zu vergleichen ist selbstverständlich. Möglicherweise erschließt sich der erst genannte Prozeß erst 
durch wiederholtes Lesen, aber es ist unbestreitbar, daß die erste Erzählung von einer Präsenz der zweiten durchdrungen ist, obwohl dies erst später ganz deutlich wird.

Ich möchte hier nur ein kurzes Beispiel hervorheben, das uns auch auf ein ständiges Merkmal des Fiktiven in der ersten Erzählung aufmerksam machen kann. Der Erzähler versucht nicht, seine Geschichte glaubwürdig zu machen. Er distanziert sich von den erzählten Ereignissen und gibt sie immer als literarisches Material an.

Zudem war unsre Held ein leichtsinniger, närrischer Junge. Wenn er jemandem begegnete, der kein Geld hatte, so bot er ihm welches an. In Bezug auf diesen Punkt bin ich indessen nicht ganz im klaren, denn aus den Werken der Schriftsteller jener Zeit kann ich durchaus nicht ersehen, daß die damaligen Menschen jemals auch Geld benötigt hätten, denn die Leute aus der guten alten Zeit führten Krieg, zogen auf Abenteuer aus, spielten die Laute unter dem Fenster der Angebeteten, trugen Achselbänder in den Farben derselben und übten den Bedrückten gegenüber stets die größten Wohlthaten, warfen das Geld mit vollen Händen auf die Straße, - doch woher sie es nahmen, darüber kann ich niergends Aufschluß erhalten. (S. 18-19)

Wo die Leute ihr Geld her haben? - lautet die Frage des Erzählers an die erste Geschichte. Diese Frage ist aber für die Welt dieser Geschichte fremd, sie ist die Hauptfrage der Welt der zweiten und organisiert dort fast alle Handlungselemente. Der Erzähler sieht die erste Geschichte von dem Gesichtspunkt der zweiten aus an. Andrerseits regt sich bei ihm Zweifel, ob das damalige Leben durch die damalige Literatur erreicht werden kann. Aber er kennt keinen anderen Weg. Folglich beziehen sich die Vorbehalte, die er in seinen Reflexionen äußert, nicht auf die Leute, sondern auf die Literatur von damals. Oder der Erzähler nimmt als Werkhypothese an, daß die damalige Literatur die damalige Wirklichkeit treu wiedergibt, und das ermöglicht ihm, sich auf das damalige Leben zu beziehen, aber er deutet ständig an, daß dieses Leben für ihn nur durch die Literatur existent wird und daß die Annahme der Homogenität der damaligen Welt bzw. ihres in der Literatur dargestellten Bildes zu komischen Ergebnissen führt. Dann aber können diese Welten auch als literarische Gattungen oder Richtungen aufgefaßt werden. Ein philologisches Indiz für diese Auffassung ist, daß die erste Ausarbeitung der ersten Erzählung, die nur einen Teil der Geschichte enthielt, eine literarische Stilparodie war. Ihr erster Satz hieß: „Sie hassen Zola und haben die Romantiker gern. “8 Die Richtungen können wir danach als Naturalismus und zeitgenössisch aufgefaßte Romantik (Romantizismus, wie es bei Mikszáth heißt) identifizieren.

Die Geschichten lesen sich nicht allein in den narratorischen Kommentaren, sondern auch in dem metaphorischen System, das einen gemeinsamen Subtext beider Geschichten liefert und das auch von dem Titel akzentuiert wird. 
Zwei leidenschaftliche Blumengärtner sind die Helden der ersten Erzählung. Der eine hat ein Gartenhaus, das der andere Käfig nennt. „Und was hältst Du wohl in diesem Käfig?" - fragt Balduin. Im Gartenhaus lebt Esre, die Pflegetochter und Flamme des Albertus. Er will davon nichts sagen, und gibt eine ausweichende Antwort: „Ja, das ist ein Geheimnis. In diesen Pavillon setze ich selbst nie einen Fuß. In diesem Käfig, mein Freund, befindet sich eine Taube. ${ }^{9}$ Doch wir wollen nicht weiter davon sprechen. “(S. 6) Nach einigen komischen Mißverständnissen lassen Balduin und Esre Albertus allein, der nach einer Periode von Leid und Mißgeschick als armer Kerl Balduins Haus erreicht. Dort sieht er ein Gartenhaus, das dem seinen von früher ähnlich ist. „Dieser Käfig hat Dich so erschreckt? lächelte Balduin. - Was gibt es denn daran zu staunen? Er gleicht ja vollkommen dem Deinigen, und enthält gleichfalls eine Taube“. (S. 41) Auch in diesem Gartenhaus lebt Esre, die Balduin nicht geheiratet hat, weil er noch rechtzeitig draufkam, daß sie Albertus liebt.

Der Titel macht den Taubensubtext auffallender. Von diesem Gesichtspunkt aus betrachtet scheint die Bewegung der Geschichte zirkulär zu sein: in der Ausgangssituation ist die Taube im Käfig und ist die Verlobte des Albertus. Die Situation am Ende ist dieselbe. Zwischen diesen Punkten sehen wir die Taube nicht. Nachträglich erfahren wir aber, daß sie nur eine ganz kurze Zeit außerhalb des Käfigs verbracht hatte, dann sowohl den Käfig, als auch Albertus zurückforderte. Die Erzählung folgte Albertus, dessen Weg aus der Welt des Gartens, des Wohlstandes und der Geborgenheit in die Welt des Elends, des Kerkers und des Ausgeliefertseins führt. Am Beginn und Ende der Geschichte sind die drei Hauptpersonen zusammen, und dieser Zustand ist harmonisch; in der Mitte bleibt einer von ihnen allein, und dieses Geteiltsein ist schlecht für jeden. Der Käfig gehört zu dem harmonischen Zustand, und ist das Symbol der Sicherheit: er steht im mit einer Mauer umgebenen Garten, und die Männer treten nicht hinein.

Diese zirkuläre metaphorische Struktur wiederholt sich in der zweiten Erzählung, doch ihre Elemente gewinnen neue Bedeutung. Diese Metaphorik ist eingentlich nur dadurch so auffallend in der zweiten Erzählung, daß sie schon aus der ersten bekannt ist.

Als Korláthy den Pflegevater der Verlobten seines Freundes um Erlaubnis bittet, dem Mädchen den Hof machen zu dürfen, antwortet dieser:

Du könntest am Ende noch den Verdacht in mir regen machen, daß Du jenem Täubchen dort eine Falle legen willst. Allein der Käfig, in dem sich die Taube befindet, gehört mir, und die Taube selbst dem Stephan. Hm! Aber unmöglich ist nichts. Hm. Was für eine Provision würdest Du mir denn geben, Frater, hehehe? (S. 68)

Der Käfig, der in der ersten Geschichte der Sicherheit der Taube diente und über dessen Schwelle dort niemand trat, scheint hier das Symbol der Herrschaft 
über die Taube zu sein. Der Pflegevater, der Besitzer des Käfigs, gibt die Taube, wem er will, oder wer ihm mehr bietet. Die Taube im Käfig scheint aller Welt ausgeliefert zu sein. Esre, die Heldin der ersten Erzählung durfte über ihr Schicksal entscheiden; Eszter ist nur das Objekt von ihr unabhängiger Männermanipulationen.

Korláthy entführt Eszter, der Pflegevater unterschlägt den Großteil ihrer Erbschaft. Korláthy, nachdem er den Rest vergeudet hat, haut ab und läßt die Frau ohne einen Heller zurück. Sie, um ihm nachreisen zu können, fälscht auf den Namen ihres Pflegevaters einen Wechsel, fährt nach Hamburg, doch einstweilen umsonst. Da ihr das Geld ausgeht, muß sie ihr Dasein als Blumenmädchen fristen. Dann aber trifft sie durch Zufall doch noch ihren Mann, den gerade eine reiche Amerikanerin aushält, und wird von diesem wegen der Wechselfälschung angezeigt. Zum Schluß der Erzählung sehen wir Korláthys Freund Altorjay in Budapest in seinem Klub wieder, wo er sich nach der „schönen Frau Korláthy“ erkundigt:

„Das Täubchen sitzt jetzt im Käfig“, bemerkte de Obergespan Gravinczy.

„Wie das?"

„Man verhaftete sie in Hamburg in der bewußten Wechselfälschungsgeschichte." (S. 108)

Die Situation „Die Taube im Käfig“ bildet den Rahmen auch in der zweiten Erzählung. Zu Beginn waren die drei Hauptpersonen zusammen, und vielleicht können wir diese Situation mit Recht harmonisch nennen. Allerdings ist es klar, daß diese Anfangssituation auch hier der Welt des Wohlstands angehört, von wo aus uns der Erzähler im mittleren Teil in die Welt des Elends führt, und im letzten Abschnitt wieder hinauf zur Elite, nähmlich in den Klub, den wir ja schon kennen. Die drei sind auch hier zusammen, wenn auch nur virtuell, nähmlich Korláthy und Altorjay in zwei verschiedenen Räumen desselben Gebäudes und Eszter als Nachricht ebenda. Sie ist physisch abwesend und aus der Welt des Wohlstandes endgültig ausgeschlossen. Ihre Abschiede und Begegnungen sind hier viel komplizierter strukturiert als in der ersten Erzählung, können aber durch ein vereinfachtes Schema beschrieben werden, das mit dem der ersten Erzählung identisch ist: Am Anfang und am Ende sieht der Erzähler die drei zusammen in der Welt des Wohlstands. Zwischen diesen beiden Endpunkten aber begleitet er jedesmal nur eine Person auf derem Weg in das Elend, was in beiden Fällen auf einen schweren Konflikt mit dem Gesetz hinausläuft. Diese strukturelle Ähnlichkeit wird durch den Die-Taube-im-Käfig-Subtext sichtbar bzw. von ihm geschaffen.

Es wurde schon gesagt, daß die beiden Geschichten in den Kommentaren der Erzähler einander lesen, und auch in den Bedeutungsänderungen der Metaphorik, wo sich genau die Entfernung von der Welt der jeweils anderen Geschichte in der 
unterschiedlichen Bedeutung desselben Wortes manifestiert. Das kann man aber auch an den Bedeutungsänderungen etlicher Begriffe beobachten.

Das auffallendste Beispiel (und das einzige, das wir hier behandeln dürfen) ist die Freundschaft. Beide Geschichten beginnen mit der Auslegung des Begriffs der Freundschaft. Balduin beschreibt seine Beziehung zu Albertus wie folgt: „Nein, gesehen haben wir uns noch niemals. Allein er schrieb ein Buch über die Blumen und ich auch, und seither stehen wir in fortwährender Korrespondenz mit einander, so daß wir die besten Freunde geworden sind.“ Der Erzähler fügt nur hinzu: „Ja, vor vierhundert Jahren gab es noch derartige Freundschaften.“ (S. 4) Es bedeutet, daß es heutzutage keine solche Freudschaft gibt, also es andersartige gibt. Der Erzähler kommentiert die erste Geschichte von dem Gesichtspunkt der zweiten aus, und er meint, die Eigenart dieser Freundschaft sei das, was ihm in der Welt der ersten Geschichte fremd ist. Erst beim Lesen der zweiten Geschichte wird es klar, warum, und was die Freundschaft in der anderen Welt bedeutet. Dort definiert der Erzähler die Freundschaft gleich am Anfang:

Im übrigen sind die beiden die besten Freunde, was sich nicht in
überströmenden Worten und Thaten kundgibt, wie in der vorange-
gangenen einfältigen Erzählung, denn Worte verhallen und Thaten
werden vergessen; doch will scripta manent, - sind ihre Namen an
vielen Stellen neben einander zu sehen, nähmlich auf Wechseln, bald
der eine, bald der andere zu oberst. (S. 53)

Der Erzähler definiert den Begriff Freundschaft in der Welt der zweiten Geschichte im Vergleich zu dem in der ersten. Der Ausdruck die besten Freunde bringt die Welt der ersten Erzählung mit und mutet an wie ein Fremdkörper im Leib der zweiten. Es ist als wollte der Erzähler die Beziehung zweier Personen in einer Fremdsprache formulieren. (Die erste Geschichte liest die zweite.) Deshalb fängt der Erzähler sofort an, den Ausdruck umzudeuten, was aber zugleich Wertung und Deutung der ersten Erzählung ist. (Die zweite Geschichte liest die erste.) Diese Deutung aber, nähmlich die neue Bedeutung, die er dem Ausdruck verleiht, ist offenbar ironisch gemeint: als ob das Wort in der Welt der zweiten Geschichte trotzdem gebräuchlich wäre, doch diesen Gebrauch der Erzähler für unvertretbar hielte. (Die erste Geschichte liest die zweite.)

Die Freundschaft ist wirklich ein Schlüsselbegriff der ersten Erzählung: das Wort Freund und seine Derivate kommen 25 mal auf den 22 Seiten der kritischen Ausgabe vor. (In der zweiten nur 4 mal auf 36 Seiten.) Als ob die ganze erste Geschichte eine Illustration zur Freundschaft wäre wie sie vor 400 Jahren war, mit (manchmal höchst parodistischen) Sätzen wie: „Ich habe gegen Dich gesündigt, als ich auch nur eine Sekunde lang an Deiner Freundschaft zu zweifeln wagte" (S. 14) und „Erst jetzt bist Du Deines Freundes in Wahrheit würdig geworden“ (S. 38). Das Wort Freund wird gewissermaßen zum Emblem der ersten Geschich- 
te, dessen seltenes Vorkommen in der zweiten auch ohne Hilfe des Erzählers die Welt der ersten heraufbeschwören kann.

Es gibt ein anderes Wort für die freundliche Beziehung in der zweiten Erzählung, das ich mit Spezi zu übersetzen versuche. Könnte Spezi vielleicht das geeignete Wort in dieser Welt sein und Freund seine Metapher, sofern es aus einer anderen Welt hierherkommt? In einer Szene muß Korláthy, von Altorjay zum Duell herausgefordert, sein Tarockspiel unterbrechen. Die Partner beginnen darüber nachzusinnen, was es für das Spiel bedeuten soll, daß er, nachdem er einen Ultimo angekündigt hat, den Spieltisch verlässt. „Die Spezis interssierte es nicht, was aus dem Kollegen werden sollte; was aus dem Ultimo werden soll, war eine weit wichtigere Frage. " ${ }^{10}$ Dieser in Klammern gesetzte Kommentar des Erzählers kann ironisch aufgefaßt werden, aber auch die Definition des Wortes Spezi darstellen: Die Spezis sind Personen, die sich oft treffen und sich in der Regel freundlich verhalten, die sich aber (und hier steckt der Unterschied zwischen Spezi und Freund!) nicht darum kümmern, wie es dem anderen ergeht. Von diesem Gesichtspunkt aus wird das einzige Vorkommen des Wortes in der erster Erzählung so vielsagend:

\begin{abstract}
Nach den in scharlachrote Gewänder gehüllten Henkern kamen zwei Gefängniswächter mit schwarzen Fahnen, denen die Verurteilten vorangetragen wurden. Auf den schwarzen Flächen der Fahnen sind je sieben flatternde Raben in blutroter Farbe zu sehen, die wohl ein Sinnbild der Verurteilten darstellen sollen. Sieben Raben! Sieben Spezis, mit denen sie noch in nähere Berührung kommen sollen. ${ }^{11}$
\end{abstract}

In der ersten Erzählung waren die Verbrecher und die Raben Spezis, in der zweiten waren es die Glieder der Hautevolee. Auch dieser Zusammenhang ist dialogisch: beide Geschichten lesen einander unter der gleichen Nennung zwei verschiedener Beziehungen. Ist das eine Metapher? Zwei verschiedene Dinge unter demselben Namen genannt. Beide Welten scheinen aber, jede für sich, die für sie gegebene Bedeutung wortwörtlich zu nehmen. Die Spannung entsteht, wenn die Welten nebeneinander kommen und der Unterschied zwischen den Bedeutungen desselben Namens sichtbar wird. Von dem Gesichtspunkt der ersten Erzählung aus betrachtet wird das Wort Spezi in der zweiten metaphorisch gebraucht, von dem der zweiten aus ist es in der ersten der Fall. An dieser Stelle schafft der Diskurs des Erzählers die Spannung, er allein ist es, der die Bedeutungen einander gegenüber stellt, zitiert aber das Wort offensichtlich aus der Umgangssprache der gegebenen Welt.

Der Dialog der Geschichten, durch Erzählen hörbar, durchdringt das ganze Werk. Am meisten fällt er auf in den narratorischen Kommentaren zu den Ereignissen, die sich von dem Gesichtspunkt der jeweiligen anderen Geschichte aus lesen, ist aber tiefsinniger in jenen Metaphern, welche sich durch beide Erzählun- 
gen ziehen und deren gemeinsamen Subtext bilden. Die Bedeutung der Metapher ändert sich grundlegend im neuen Kontext, und der Leser kann in den Änderungen die grundlegenden Unterschiede zwischen diesen Geschichten, zwischen diesen Welten erkennen. Die Tatsache, daß die Betonung auf den Unterschieden legt, widerspricht keineswegs meinem Ausgangspunkt, daß die Beziehung der Geschichten eine metaphorische sei. Denn nicht nur auf Ähnlichkeiten basiert die Metapher. Wenn der Erzähler behauptete, die Geschichten seien identisch, gab er dem Leser die Aufgabe, die Ähnlichkeiten und Gleichheiten zu finden. Aber die Gleichheiten haben auch die Unterschiede sichtbar gemacht. Die Äußerung, daß wir hier eine Metapher haben, machte die Geschichten kommensurabel, die sonst kaum etwas miteinander zu tun hätten. Nachdem wir erkannt hatten, daß die Metapher der Taube beide Geschichten auf dieselbe Weise strukturiert, müßen wir auch erkennen, wie sehr verschieden die Metapher selbst in jeder Geschichte und wie sehr verschieden jede Geschichte trotz der gemeinsamen metaphorischen Struktur ist. Wenn wir beobachten, welch intensiven Diskurs die Personen und Erzähler über einige Zentralbegriffe (wie z.B. Freundschaft) führen, werden wir unbedingt auch entdecken, welch verschiedene Bedeutungen diese Begriffe in den verschiedenen Kontexten haben. Dieser Prozeß ist insoweit metaphorisch, daß beide Geschichten einander gegenseitig mit dem Gesichtspunkt versehen, von dem aus diese Fremdheit wahrgenommen und beurteilt werden kann.

\section{Anmerkungen}

1. Die wichtigsten Initiativen dieser Richtung waren: I. A. Richards, The Philosophy of Rhetoric, Oxford 1936; M. Black, Models and Metaphors, Ithaca 1962.

2. P. K. Garrett, The Victorian Multiplot Novel: Studies in Dialogical Form, New Heaven-London 1980, 9.

3. Die Zitaten - wenn nur mit Nummer der Seite angegeben - folgen Ludwig Wechslers Übersetzung: Koloman Mikszáth, Die Taube im Käfig: Zwei Geschichten in einer, Berlin-Leipzig o.J.

4. Richards, a. a. O. 96.

5. Black, a. a. O. 44.

6. Meine Übersetzung: M. M. Bakhtin, Discourse in the Novel. In: The Dialogic Imagination. Austin-London 1981, 277.

7. F. R. Ankersmit, Narrative Logic: A Semantic Analysis of the Historian's Language. The HagueBoston-London 1983, 209-220.

8. Mikszáth Kálmán összes müvei 4,157:1, meine Übersetzung.

9. Die Bedeutung des Ausdrucks „,meine Taube“ auf Ungarisch ist dem deutschen „mein Liebchen" ähnlich.

10. S. 78, wo aber statt Spezis Kameraden steht.

11. S. 29, wo aber statt Spezis ,,gute Freunde "steht. 